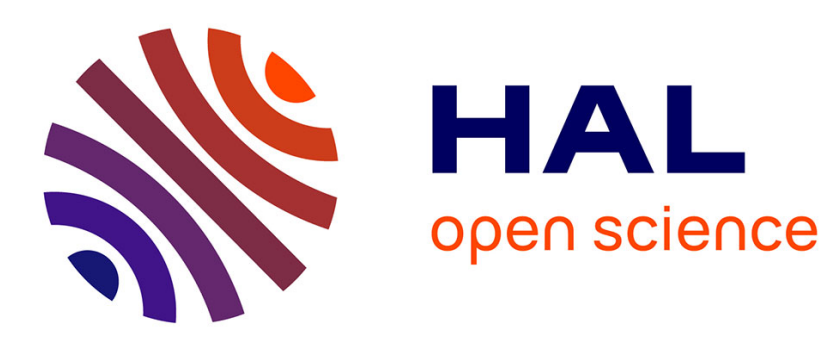

\title{
Linear viscoelasticity of carbosilane dendrimer melts
}

\author{
Maxim Dolgushev, Denis A Markelov, Erkki Lähderanta
}

\section{To cite this version:}

Maxim Dolgushev, Denis A Markelov, Erkki Lähderanta. Linear viscoelasticity of carbosilane dendrimer melts. Macromolecules, 2019, 52 (6), pp.2542-2547. 10.1021/acs.macromol.8b02250 . hal02188679

\section{HAL Id: hal-02188679 \\ https://hal.sorbonne-universite.fr/hal-02188679}

Submitted on 18 Jul 2019

HAL is a multi-disciplinary open access archive for the deposit and dissemination of scientific research documents, whether they are published or not. The documents may come from teaching and research institutions in France or abroad, or from public or private research centers.
L'archive ouverte pluridisciplinaire HAL, est destinée au dépôt et à la diffusion de documents scientifiques de niveau recherche, publiés ou non, émanant des établissements d'enseignement et de recherche français ou étrangers, des laboratoires publics ou privés. 


\title{
Linear viscoelasticity of carbosilane dendrimer melts
}

\author{
Maxim Dolgushev, ${ }^{*, \dagger}$ Denis A. Markelov, ${ }^{\ddagger}$ and Erkki Lähderanta ${ }^{\mathbb{I l}}$ \\ †Sorbonne Université, CNRS, Laboratoire de Physique Théorique de la Matière Condensée, \\ LPTMC, F-75005 Paris, France \\ $\ddagger$ St. Petersburg State University, 7/9 Universitetskaya nab., St. Petersburg, 199034, Russia \\ I[Department of Physics, Lappeenranta University of Technology, Lappeenranta, Finland \\ E-mail: dolgushev@lptmc.jussieu.fr
}

\begin{abstract}
We report on shear-stress relaxation of melts of poly(butylcarbosilane) dendrimers. The system is studied by means of the molecular dynamics simulations with atomistic resolution. The key investigated quantities are the dynamical shear-stress relaxation modulus and - its counterparts in the frequency domain - the storage and loss moduli. We show the existence of three main characteristic regimes of the mechanical relaxation (going from high to low frequencies): (i) relaxation of tension related to the finite extensibility of the macromolecules; (ii) relaxation related to the dendritic architecture (inner and branch relaxation); (iii) mobility of the dendrimer as a whole. The tension relaxation is independent of the dendrimers' size and leads to a power law characterized by an exponent 0.7 . The processes at low frequencies (branch relaxation and dendrimer mobility as a whole) strongly depend on the molecular mass and do not lead to a universal behavior.
\end{abstract}

Dendrimers are extraordinary macromolecules with a perfectly symmetric treelike structure. ${ }^{[1]}$ While being synthesized first in the seventies, ${ }^{[2]}$ nowadays they enjoy a growing attention. ${ }^{[3,4]}$ The reason for this lies not only in the appearance of new types of these macromolecules ${ }^{[5]}$ and in their possible applications (as nanocarriers, ${ }^{[6]}$ rheology modifiers, ${ }^{[7]}$ nanoscale catalysts, ${ }^{[8]}$ to 

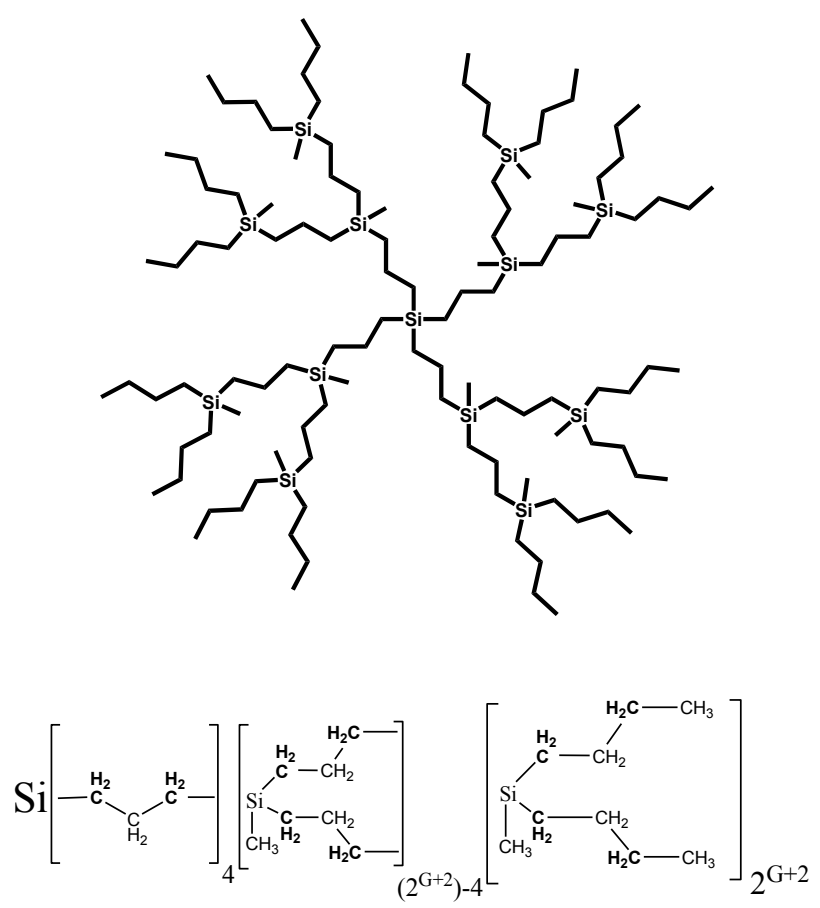

Figure 1: The structure of poly(butylcarbosilane) dendrimers (CSD). Top figure shows a CSD of generation $G=2$.

name only a few), but also in success of experimental techniques in studying physical properties of dendrimers. ${ }^{\left[{ }^{-12]}\right.}$ Unlike linear chains that almost entirely consist of inner groups, roughly half of the dendrimers' segments are terminal. This fact leads to sensitivity of the dendrimers' properties to their chemistry. ${ }^{[13]}$ Therefore full-atomistic approaches may build a solid bridge between theory and experiment. Carbosilane dendrimers (CSD), ${ }^{[14,15]}$ Figure 1, turn out to be very suitable compounds for such studies. They do not contain specific fragments, such as charged groups or stiff and massive segments. This enables one to focus investigations on the role of dendritic architecture. In particular, the dendritic architecture is reflected in the relaxation times that can be associated with the inner relaxation as well as with the relaxation of (sub)branches as whole, in agreement with qualitative predictions of basic theory. ${ }^{[16,17]}$ Given that the overall relaxation of dendrimer's (sub)branches depend on their molecular mass, the spectrum of the corresponding relaxation times in case of dendrimers is very broad. ${ }^{[18]}$ Undoubtedly these findings are very important also for the rheological properties of dendrimers, although the broadness of the dendrimers' spectra requires very extensive simulations. Here it is worth mentioning that in case of linear chains, after molecular 


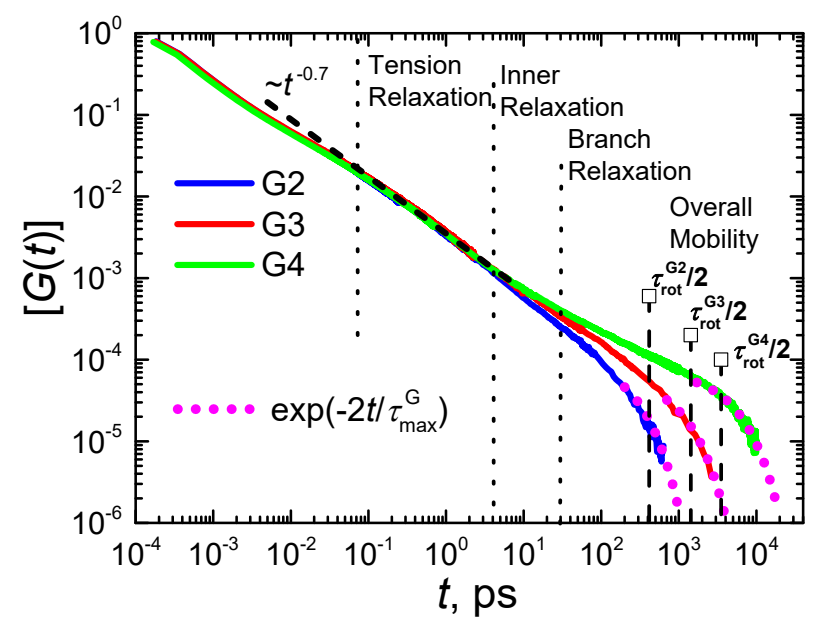

Figure 2: Double-logarithmic representation of the normalized shear-stress relaxation modulus $[G(t)] \equiv G(t) / G(0)$ for melts of CSD at generation $G=2,3,4$. Dashed line indicates the power law associated with the tension relaxation, dotted lines illustrate the exponential tail of the moduli. Also we show the position of the rotational relaxation times $\tau_{\text {rot }}$ (column $\tau_{T}=0.5$ in Table 1) and indicate regions related to different processes, see text for details. The time scale is given in ps unit of the GROMACS package with the parameter $\tau_{T}=0.5$ of the Langevin thermostat.

dynamics (MD) computations of typical dynamic quantities were performed, it took more than one decade to study the mechanical relaxation. This is because the calculation of the stress-stress relaxation needs to bridge all processes appearing in the system, see. ${ }^{[19]}$ Full-atomistic simulations of CSD melts have been performed very recently in works. ${ }^{[16,20,21]}$ Dendrimer melts with other chemistry were simulated in Ref. ${ }^{[22,23]}$ and the coarse-grained non-equilibrium MD simulations were used for studies of the shear viscosity of dendrimer melts ${ }^{[24,25]}$ and of the rheological properties of dendrimer melts undergoing planar elongational flow. ${ }^{[26]}$ None of these studies investigated the dynamical shear-stress relaxation modulus. Our work presents, to the best of our knowledge, the first theoretical study of the shear-stress relaxation of CSD melts. The main results are summarized in Figure 2. It shows at short times a universal behavior of the shear-stress relaxation modulus for all systems and at longer times a clear discrepancy for dendrimers of different generation.

The dendrimers of generation $G=2,3,4$ (in short: G2, G3, and G4 dendrimers, respectively) in a melt are studied by MD simulations employing the GROMACS package ${ }^{\text {[27] }}$ (with Verlet scheme ${ }^{[28]}$. Each simulation box with periodic boundaries contained 27 macromolecules. All interactions are considered in the united atoms model within Gromos53a6 force-field. ${ }^{[29]}$ The 

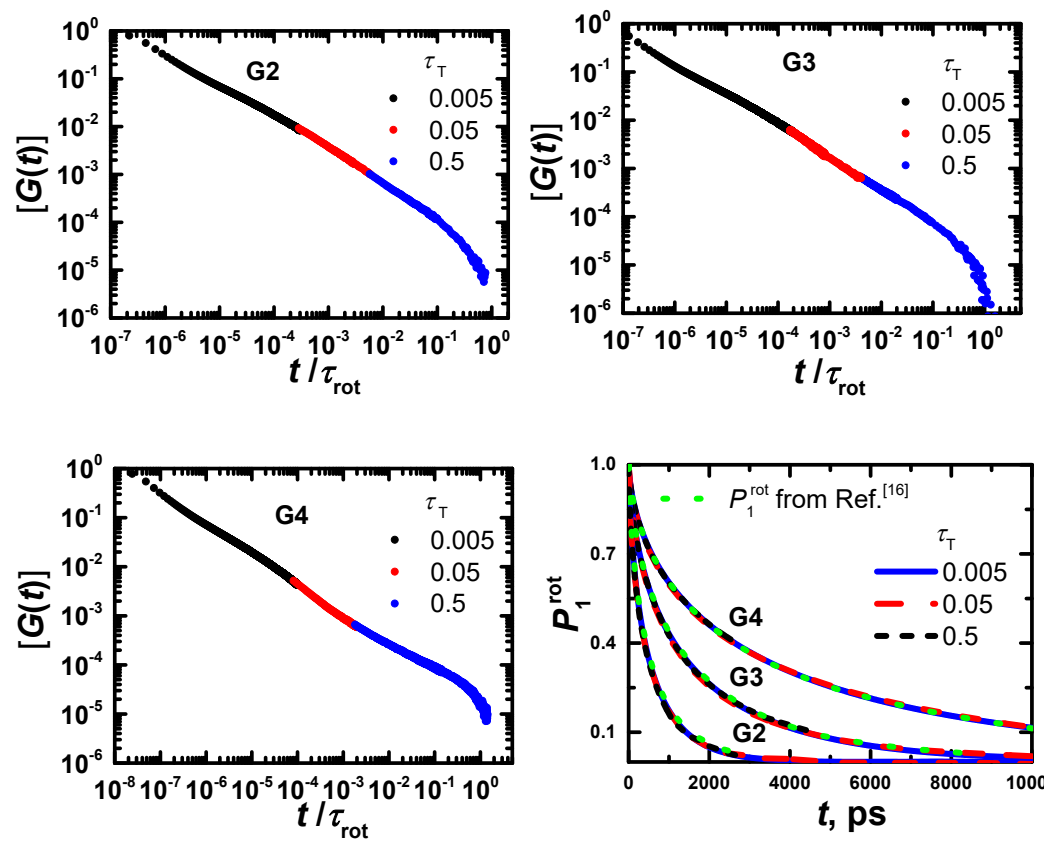

Figure 3: The normalized shear-stress relaxation modulus $[G(t)] \equiv G(t) / G(0)$ for a melt of CSD (G2, G3, and G4), calculated from simulations employing Langevin thermostat with different values of the parameter $\tau_{T}$. The curves are rescaled with $\tau_{\text {rot }}$ (Table 1 ) that are obtained from the rotational autocorrelation function $P_{1}^{\text {rot }}(t)$ (the right bottom subfigure, here the time unit is as in Figure 2).

systems have been kept with Langevin thermostat at temperature $600 \mathrm{~K}$ with different values of coupling constant $\tau_{\mathrm{T}}: 0.005,0.05,0.5 \mathrm{ps}$. The variation of $\tau_{\mathrm{T}}$ that is related to the friction allows us to control the initial oscillations of the shear-stress relaxation modulus. In order to achieve better statistics we simulated ten replicas for the highest value of the parameter $\tau_{T}$. First, each system (whose initial configurations were obtained from the final frames of the trajectories of Ref. ${ }^{[16]}$ ) was equilibrated in the NPT ensemble with Berendsen barostat ${ }^{[30]}$ (G2 and G3 during 10 ns and G4 during $30 \mathrm{~ns}$ ). Then the equilibration of the systems was proceeded in the NVT ensemble (the duration for G2: $0.1 \mu \mathrm{s}$, for G3: $0.2 \mu \mathrm{s}$, and for G4: $0.6 \mu \mathrm{s}$ ). For the analysis, successive $0.6 \mu \mathrm{s}, 1 \mu \mathrm{s}$, and $2.1 \mu \mathrm{s}$ of the trajectories for $\mathrm{G} 2, \mathrm{G} 3$, and $\mathrm{G} 4$ systems, respectively, were used for calculation of the physical characteristics of the systems.

The shear-stress relaxation is calculated based on the stress tensor $\hat{P}$,

$$
\hat{P}=\frac{1}{V}\left(\sum_{i} m_{i} \vec{v}_{i} \otimes \vec{v}_{i}+\sum_{i<j} \vec{r}_{i j} \otimes \vec{F}_{i j}\right)
$$


Table 1: Rotational relaxation times $\tau_{\text {rot }}$ for CSD of different generation $G$ for different values of the parameter $\tau_{T}$ characterizing the Langevin thermostat. Also $\tau_{\text {rot }}$ from Ref. ${ }^{[16]}$ (V-rescale thermostat and NPT ensemble) are shown. The time units are ps of the GROMACS package.

\begin{tabular}{c|cccc}
\hline$G$ & $\tau_{T}=0.005$ & $\tau_{T}=0.05$ & $\tau_{T}=0.5$ & V-rescale $^{[16]}$ \\
\hline 2 & 45393 & 5404 & 831 & 540 \\
3 & 154540 & 18400 & 2830 & 1800 \\
4 & 416000 & 45700 & 7033 & 4470 \\
\hline
\end{tabular}

where $m_{i}$ and $\vec{v}_{i}$ are the mass and the velocity of the united atom group $i, \vec{F}_{i j}$ is the force exerted from $j$ to $i, \vec{r}_{i j}$ is the distance vector between them, and $V$ is the box volume. The dynamic shear-stress modulus $G(t)$ is determined based on the stress tensor, ${ }^{[31-33]}$

$$
G(t)=\frac{V}{30 k_{B} T} \sum_{(\alpha \beta)}\left(6\left\langle P_{\alpha \beta}(t) P_{\alpha \beta}(0)\right\rangle+\left\langle N_{\alpha \beta}(t) N_{\alpha \beta}(0)\right\rangle\right)
$$

where the sum runs over the pairs $(\alpha \beta)=x y, y z, z x$ and $N_{\alpha \beta}=P_{\alpha \alpha}-P_{\beta \beta}$. The simulations performed at different friction are bridged based on the rotational autocorrelation function

$$
P_{1}^{\mathrm{rot}}(t)=\langle\mathbf{u}(t) \cdot \mathbf{u}(0)\rangle
$$

where $\mathbf{u}(t)$ is the unit vector at time $t$ directed from the silicone atom in the core to a silicone atom of the terminal layer. Thus, this function characterizes the overall rotation of the dendrimer molecule. From the exponential tail of $P_{1}^{\text {rot }}(t)$ (that is observed in the half-logarithmic scales) we have deduced the rotational relaxation times $\tau_{\text {rot }}$, see Table 1. As can be seen in Figure 3 (bottom right), the functions $P_{1}^{\text {rot }}(t)$ could be perfectly superimposed when different coupling constants of the thermostat are considered, if one takes $\tau_{\text {rot }}$ as the benchmark time. To make a connection to the previous work $^{[16]}$ (where a different ensemble (NPT) and a different thermostat (V-rescale) were used), we show also those functions $P_{1}^{\text {rot }}(t)$ in Figure 3 and provide corresponding values of $\tau_{\text {rot }}$ in Table 1. Rescaling the time with $\tau_{\text {rot }}$, we obtain very smooth overlap of $G(t)$, see Figure 3 . The ensuing curves are collected in Figure 2.

Now, direct comparison of $G(t)$ for CSD of generations $G=2,3,4$, Figure 2, shows that at short 


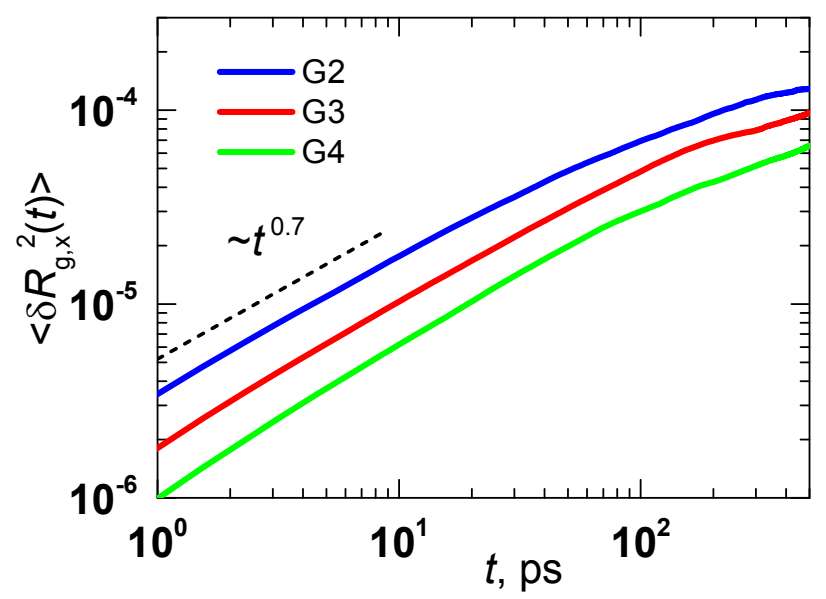

Figure 4: Fluctuations of the $x$-component of the gyration tensor $\left\langle\delta R_{g, x}^{2}(t)\right\rangle$ of CSD at different generation. The power law $t^{0.7}$ is also indicated. The time unit is as in Figure 2.

times the moduli overlap each other, whereas at longer times one gets a slower stress relaxation for larger systems. It is known from the basic (bead-spring) theory ${ }^{[18,34-36]}$ that the relaxation spectrum of dendrimers differs qualitatively from that of the linear chains. Namely, dendrimers have two types of modes: spatially periodic and spatially exponential. The first type of modes leads to the relaxation times of the so-called inner spectrum ${ }^{[17,35]}$ that weakly depends on the dendrimers' generation. The inner spectrum is rather narrow and the corresponding (average) time is the minimal time $\tau_{\min }$ associated with the relaxation of the dendritic architecture. ${ }^{[16,17]}$ It has the same order as the time of reorientation relaxation of the terminal segments, i.e., for CSD $\tau_{\min }=1-10 \mathrm{ps}$, see Ref. ${ }^{[16]}$ At this time the $G(t)$-curves start to separate for dendrimers of different generations. We remark that the modes corresponding to the inner spectrum involve the motion of all beads (with spatially periodic phases $)^{[18,34-36]}$ and therefore the inner relaxation is not the relaxation of individual segements. The spatially exponential modes are related to the relaxation of (sub)branches as whole that build branch relaxation spectrum. ${ }^{[18,35,36]}$ The corresponding relaxation times depend on the size of the subbranches whose molecular weight grows exponentially with generation. We note that there are no power-law behaviors for the relaxation times and for the corresponding dynamic quantities in the bead-spring theory of dendrimers. ${ }^{[34,35]}$ This is another point that distinguishes dendrimers from the linear chains. In addition to these features, the simulations of dendrimers ${ }^{[17,37]}$ have suggested 
that at long times the theory has to be adjusted by a proper description of overall mobility. In particular, the bead-spring theory does not describe properly the rotational degrees of freedom of the whole macromolecule. ${ }^{[38]}$ As can be seen in Figure 2, going to longer times $\left(t>\tau_{\text {min }}\right)$, one does not find any universal scaling of $G(t)$. The relaxation is dominated by the dendrimers' architecture and the presence of larger branches (whose molecular mass grows exponentially with generation) strongly slows down the dynamics of the larger dendrimers. At longest times, the moduli $G(t)$ show a typical exponential tail, ${ }^{[39]} G(t) \propto \exp \left(-2 t / \tau_{\max }\right)$. Replotting $G(t)$ in half-logarithmic scales we find the maximal times $\tau_{\max }=461,1980$, and 9600 (ps of GROMACS for the Langevin thermostat at $\tau_{T}=0.5$ ) for G2, G3, and G4, respectively. Comparing them with the corresponding rotational relaxation times $\tau_{\text {rot }}$ (see column $\tau_{T}=0.5$ in Table 1 ), one realizes that for G4 the stress relaxation dynamics is somewhat slower than the rotational relaxation, that indicates the presence of significant interactions between different dendrimers.

In this work, at short times, we find another point missed by the bead-spring theory that is related to the finite extensibility of dendrimer macromolecules. Namely, in Figure 2 we see a new universal behavior at short times. In line with works ${ }^{[40-42]}$ on the viscoelasticity of inextensible semiflexible filaments, this process is related to the restricted extensibility of the macromolecules and represents the corresponding relaxation of tension. There it was found that the tension relaxation of linear filaments leads to a $t^{-3 / 4}$ power law behavior of the modulus at short times. Here, for dendrimers, we find slightly different scaling exponent, close to -0.7 . In case of linear filaments the exponent $-3 / 4$ is directly related to the longitudinal fluctuation around the contraction of the end-to-end distance. Given that a dendrimer has many ends, we consider the fluctuations of the $x$-component of the gyration tensor $\left\langle\delta R_{g, x}^{2}(t)\right\rangle \equiv\left\langle\left[R_{g, x}(t)-R_{g, x}(0)\right]^{2}\right\rangle$, along which direction the macromolecule has its maximal extension, see Figure 4. Here we find also the exponent 0.7 (that is close to that $3 / 4$ for the longitudinal fluctuations of the linear filaments ${ }^{[40-43]}$ ), which we observed in the modulus $G(t)$ in Figure 2.

Finally, Figure 5 presents experimentally relevant storage $\left[G^{\prime}(\omega)\right]$ and loss $\left[G^{\prime \prime}(\omega)\right]$ moduli, which are obtained from $[G(t)]$ of Figure 2 using the Fourier transform, ${ }^{[33,44]} G^{\prime}(\omega)+\mathrm{i} G^{\prime \prime}(\omega)=$ 

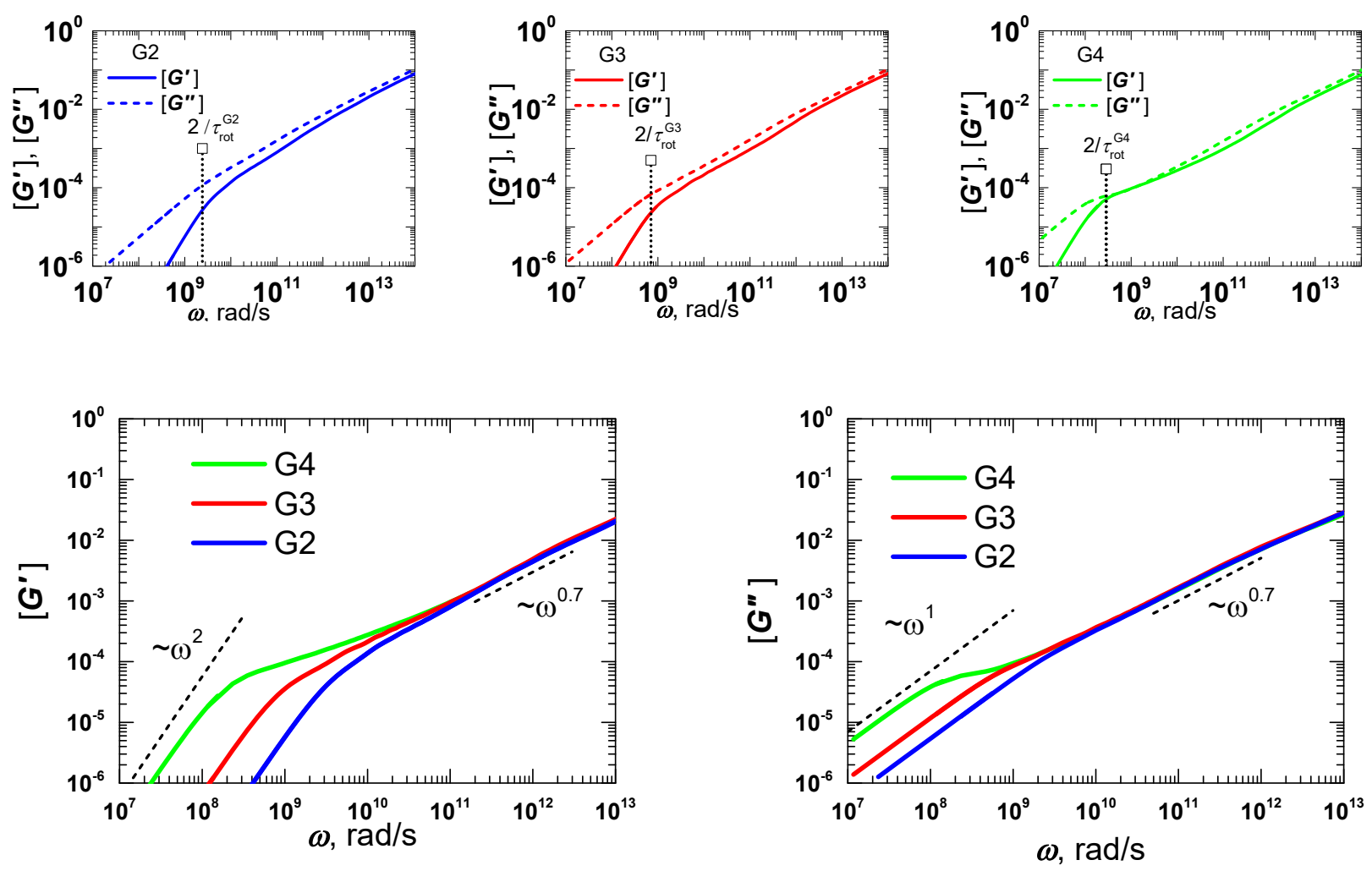

Figure 5: Double-logarithmic representation of the storage $\left[G^{\prime}(\omega)\right]$ and loss $\left[G^{\prime \prime}(\omega)\right]$ moduli for CSD melts. Upper subfigures show the moduli for G2, G3, and G4 (from left to right) and the lower ones represent the moduli for different generations at one plot. The radial frequency unit correspods to the time unit of Figure 2 .

$\mathrm{i} \omega \int G(t) e^{-\mathrm{i} \omega t} \mathrm{~d} t$. At low frequencies (smaller than the inverse rotational relaxation times, see the upper panel of Figure 5) the moduli show scalings ${ }^{[44]} G^{\prime}(\omega) \sim \omega^{2}$ and $G^{\prime \prime}(\omega) \sim \omega^{1}$. At high frequencies the scaling exponent -0.7 of $G(t)$ (see Figure 2) leads to a $G^{\prime}(\omega) \sim G^{\prime \prime}(\omega) \sim \omega^{0.7}$ behavior. Strikingly, the same power law in $G^{\prime}(\omega)$ and $G^{\prime \prime}(\omega)$ is observed experimentally for PPI dendrimer melts of generations 3 to 5 (that have similar molecular weights as CSD dendrimers G2 to G4, respectively). ${ }^{[11]}$ Moreover, the power law behavior is observed when the normalized moduli $\left[G^{\prime}(\omega)\right]$ and $\left[G^{\prime \prime}(\omega)\right]$ take values between 0.001 and $0.1^{[11]}$ that corresponds to the results of our simulations. We note also that the same exponent by the moduli at high frequencies was recently found in the experiments on melts of branched POSS macromolecules. ${ }^{[45]}$ In that work this finding was connected with the Zimm model. ${ }^{[46]}$ Here we persuade ourselves that the reason for this behavior lies rather in the relaxation of tension. Now, it is interesting to observe the relative position of $G^{\prime}(\omega)$ and $G^{\prime \prime}(\omega)$ at different generation (the upper part of Figure 5). While for G2 
and G3 $G^{\prime}(\omega)$ lies strictly below $G^{\prime \prime}(\omega)$, for G4 the curves touch each other at low frequencies just before going to the terminal regime, in line with the enhanced slowing down of $G(t)$ for

G4. If one looks at the terminal $\omega^{1}$-regime for $G^{\prime \prime}(\omega)$ (whose prefactor is related to the viscosity $\eta^{[44]}$ ) one sees a non-monotonous shift toward low frequencies with increasing generation (we get $[\eta(\mathrm{G} 3) / \eta(\mathrm{G} 2)] \approx 2.1$ and $[\eta(\mathrm{G} 4) / \eta(\mathrm{G} 2)] \approx 7.7)$. This indicates the growing contribution of the interactions between the macromolecules. Interestingly, very recent experimental works ${ }^{[47,48]}$ demonstrate that CSD melts of higher molecular masses can show a network formation. Here, at G4, however, we are still far from such effects neither from the entanglements, by which the moduli show for hyperbranched polymers a qualitatively distinct behavior. ${ }^{[49]}$ Closing the paper, we note that it would be interesting to investigate in the future how the findings of this work will resemble at higher generations of CSD and at lower temperatures.

\section{Acknowledgement}

The authors thank Joachim Wittmer (Institut Charles Sandron, CNRS, Strasbourg, France) for fruitful discussions. The simulations were performed in Computer Science Center (CSC) of Finland and in Computer Resources Center of Saint Petersburg State University. This work is supported by the the grant of researchers' mobility of Academy of Finland (No. 318608), by the European Research Council Starting Grant (No. FPTOpt-277998), and by the Russian Foundation for Basic Research (No. 19-03-00715).

\section{References}

(1) Tomalia, D. A.; Baker, H.; Dewald, J.; Hall, M.; Kallos, G.; Martin, S.; Roeck, J.; Ryder, J.; Smith, P. A new class of polymers: starburst-dendritic macromolecules. Polym. J. 1985, 17, 117. 
(2) Buhleier, E.; Wehner, W.; Vögtle, F. Cascade and Nonskid-Chain-Like Syntheses of Molecular Cavity Topologies. Synthesis 1978, 2, 155-158.

(3) Astruc, D.; Boisselier, E.; Ornelas, C. Dendrimers designed for functions: from physical, photophysical, and supramolecular properties to applications in sensing, catalysis, molecular electronics, photonics, and nanomedicine. Chem. Rev. 2010, 110, 1857-1959.

(4) Newkome, G. R.; Shreiner, C. Dendrimers derived from $1 \rightarrow 3$ branching motifs. Chem. Rev. 2010, 110, 6338-6442.

(5) Sowinska, M.; Urbanczyk-Lipkowska, Z. Advances in the chemistry of dendrimers. New J. Chem. 2014, 38, 2147-2708.

(6) Kesharwani, P.; Jain, K.; Jain, N. K. Dendrimer as nanocarrier for drug delivery. Progr. Polym. Sci. 2014, 39, 268-307.

(7) Kuang, T.; Chang, L.; Fu, D.; Yang, J.; Zhong, M.; Chen, F.; Peng, X. Improved crystallizability and processability of ultra high molecular weight polyethylene modified by poly (amido amine) dendrimers. Polym. Eng. Sci. 2017, 57, 153-160.

(8) Caminade, A.-M. Inorganic dendrimers: recent advances for catalysis, nanomaterials, and nanomedicine. Chem. Soc. Rev. 2016, 45, 5174-5186.

(9) Pinto, L. F.; Correa, J.; Martin-Pastor, M.; Riguera, R.; Fernandez-Megia, E. The dynamics of dendrimers by NMR relaxation: interpretation pitfalls. J. Am. Chem. Soc. 2013, 135, $1972-1977$.

(10) Pinto, L. F.; Riguera, R.; Fernandez-Megia, E. Stepwise filtering of the internal layers of dendrimers by transverse-relaxation-edited NMR. J. Am. Chem. Soc. 2013, 135, 1151311516.

(11) Hofmann, M.; Gainaru, C.; Cetinkaya, B.; Valiullin, R.; Fatkullin, N.; Rössler, E. A. FieldCycling Relaxometry as a Molecular Rheology Technique: Common Analysis of NMR, Shear 
Modulus and Dielectric Loss Data of Polymers vs Dendrimers. Macromolecules 2015, 48, $7521-7534$.

(12) Mohamed, F.; Hofmann, M.; Pötzschner, B.; Fatkullin, N.; Rössler, E. A. Dynamics of PPI Dendrimers: A Study by Dielectric and 2H NMR Spectroscopy and by Field-Cycling $1 \mathrm{H}$ NMR Relaxometry. Macromolecules 2015, 48, 3294-3302.

(13) Tande, B. M.; Wagner, N. J.; Kim, Y. H. Influence of end groups on dendrimer rheology and conformation. Macromolecules 2003, 36, 4619-4623.

(14) Schlenk, C.; Frey, H. Carbosilane dendrimers-synthesis, functionalization, application. Silicon Chemistry 1999, 3-14.

(15) Muzafarov, A. M.; Vasilenko, N. G.; Tatarinova, E. A.; Ignat'eva, G. M.; Myakushev, V. M.; Obrezkova, M. A.; Meshkov, I. B.; Voronina, N. V.; Novozhilov, O. V. Macromolecular nano-objects as a promising direction of polymer chemistry. Polym. Sci. Ser. C 2011, 53, 48.

(16) Markelov, D. A.; Shishkin, A. N.; Matveev, V. V.; Penkova, A. V.; Lähderanta, E.; Chizhik, V. I. Orientational Mobility in Dendrimer Melts: Molecular Dynamics Simulations. Macromolecules 2016, 49, 9247-9257.

(17) Markelov, D. A.; Dolgushev, M.; Lähderanta, E. NMR Relaxation in Dendrimers. Annu Rep. NMR Spectrosc. 2017, 91, 1-66.

(18) Fürstenberg, F.; Dolgushev, M.; Blumen, A. Analytical model for the dynamics of semiflexible dendritic polymers. J. Chem. Phys. 2012, 136, 154904.

(19) Likhtman, A. E.; Sukumaran, S. K.; Ramirez, J. Linear viscoelasticity from molecular dynamics simulation of entangled polymers. Macromolecules 2007, 40, 6748-6757.

(20) Shishkin, A. N.; Markelov, D. A.; Matveev, V. V. Molecular dynamics simulation of poly(butyl)carbosilane dendrimer melts at 600 K. Russ. Chem. Bull. 2016, 65, 67-74. 
(21) Balabaev, N. K.; Mazo, M. A.; Kramarenko, E. Y. Insight into the Structure of Polybutylcarbosilane Dendrimer Melts via Extensive Molecular Dynamics Simulations. Macromolecules 2017, 50, 432-445.

(22) Bag, S.; Jain, M.; Maiti, P. K. Charge Transport in Dendrimer Melts Using Multiscale Modeling Simulation. J. Phys. Chem. B 2016, 120, 9142-9151.

(23) Smeijers, A. F.; Markvoort, A. J.; Pieterse, K.; Hilbers, P. A. J. Coarse-grained simulations of poly(propylene imine) dendrimers in solution. J. Chem. Phys. 2016, 144, 074903.

(24) Bosko, J. T.; Todd, B. D.; Sadus, R. J. Viscoelastic properties of dendrimers in the melt from nonequlibrium molecular dynamics. J. Chem. Phys. 2004, 121, 12050-12059.

(25) Bosko, J. T.; Todd, B. D.; Sadus, R. J. Molecular simulation of dendrimers and their mixtures under shear: Comparison of isothermal-isobaric (NpT) and isothermal-isochoric (NVT) ensemble systems. J. Chem. Phys. 2005, 123, 034905.

(26) Hajizadeh, E.; Todd, B. D.; Daivis, P. J. Nonequilibrium molecular dynamics simulation of dendrimers and hyperbranched polymer melts undergoing planar elongational flow. J. Rheol. 2014, 58, 281-305.

(27) Abraham, M. J.; Murtola, T.; Schulz, R.; Páll, S.; Smith, J. C.; Hess, B.; Lindah, E. Gromacs: High performance molecular simulations through multi-level parallelism from laptops to supercomputers. SoftwareX 2015, 1-2, 19-25.

(28) Páll, S.; Hess, B. A flexible algorithm for calculating pair interactions on SIMD architectures. Comp. Phys. Comm. 2013, 184, 2641-2650.

(29) Oostenbrink, C.; Villa, A.; Mark, A. E.; Van Gunsteren, W. F. A biomolecular force field based on the free enthalpy of hydration and solvation: the GROMOS force-field parameter sets 53A5 and 53A6. J. Comp. Chem. 2004, 25, 1656-1676. 
(30) Berendsen, H. J. C.; Postma, J. P. M.; van Gunsteren, W. F.; DiNola, A.; Haak, J. R. Molecular dynamics with coupling to an external bath. J. Chem. Phys. 1984, 81, 3684-3690.

(31) Allen, M. P.; Tildesley, D. J. Computer simulation of liquids; Oxford university press, 1989.

(32) Ramírez, J.; Sukumaran, S. K.; Vorselaars, B.; Likhtman, A. E. Efficient on the fly calculation of time correlation functions in computer simulations. J. Chem. Phys. 2010, 133, 154103.

(33) Wittmer, J. P.; Xu, H.; Benzerara, O.; Baschnagel, J. Fluctuation-dissipation relation between shear stress relaxation modulus and shear stress autocorrelation function revisited. Mol. Phys. 2015, 113, 2881-2893.

(34) Cai, C.; Chen, Z. Y. Rouse Dynamics of a Dendrimer Model in the $\Theta$ Condition. Macromolecules 1997, 30, 5104-5117.

(35) Gotlib, Y. Y.; Markelov, D. A. Theory of the Relaxation Spectrum of a Dendrimer Macromolecule. Polym. Sci. Ser. A 2002, 44, 1341-1350.

(36) Gurtovenko, A. A.; Markelov, D. A.; Gotlib, Y. Y.; Blumen, A. Dynamics of dendrimer-based polymer networks. J. Chem. Phys. 2003, 119, 7579.

(37) Markelov, D. A.; Gotlib, Y. Y.; Darinskii, A. A.; Lyulin, A. V.; Lyulin, S. V. Local orientational mobility in dendrimers. Theory and computer-aided simulation. Polym. Sci. Ser. A 2009, 51, 331-339.

(38) Kelly, J.; Grosberg, A. Y.; Bruinsma, R. Generalized Flory Theory for Rotational Symmetry Breaking of Complex Macromolecules. arXiv preprint 2018, arXiv:1809.01047.

(39) Dolgushev, M.; Wittmer, J. P.; Johner, A.; Benzerara, O.; Meyer, H.; Baschnagel, J. Marginally compact hyperbranched polymer trees. Soft Matter 2017, 13, 2499-2512.

(40) Granek, R. From semi-flexible polymers to membranes: anomalous diffusion and reptation. J. Physique II 1997, 7, 1761-1788. 
(41) Morse, D. C. Viscoelasticity of concentrated isotropic solutions of semiflexible polymers. 2. Linear response. Macromolecules 1998, 31, 7044-7067.

(42) Broedersz, C. P.; MacKintosh, F. C. Modeling semiflexible polymer networks. Rev. Mod. Phys. 2014, 86, 995.

(43) Saphiannikova, M.; Toshchevikov, V.; Gazuz, I.; Petry, F.; Westermann, S.; Heinrich, G. Multiscale approach to dynamic-mechanical analysis of unfilled rubbers. Macromolecules 2014, 47, 4813-4823.

(44) Ferry, J. D. Viscoelastic properties of polymers; John Wiley \& Sons, 1980.

(45) Liu, G.; Feng, X.; Lang, K.; Zhang, R.; Guo, D.; Yang, S.; Cheng, S. Z. Dynamics of ShapePersistent Giant Molecules: Zimm-like Melt, Elastic Plateau, and Cooperative Glass-like. Macromolecules 2017, 50, 6637-6646.

(46) Zimm, B. H. Dynamics of polymer molecules in dilute solution: viscoelasticity, flow birefringence and dielectric loss. J. Chem. Phys. 1956, 24, 269-278.

(47) Matveev, V. V.; Markelov, D. A.; Dvinskikh, S. V.; Shishkin, A. N.; Tyutyukin, K. V.; Penkova, A. V.; Tatarinova, E. A.; Ignat'eva, G. M.; Milenin, S. A. Investigation of Melts of Polybutylcarbosilane Dendrimers by 1 H NMR Spectroscopy. Sci. Rep. 2017, 7, 13710.

(48) Vasil'ev, V. G.; Kramarenko, E. Y.; Tatarinova, E. A.; Milenin, S. A.; Kalinina, A. A.; Papkov, V. S.; Muzafarov, A. M. An unprecedented jump in the viscosity of high-generation carbosilane dendrimer melts. Polymer 2018, 146, 1-5.

(49) Schubert, C.; Osterwinter, C.; Tonhauser, C.; Schömer, M.; Wilms, D.; Frey, H.; Friedrich, C. Can Hyperbranched Polymers Entangle? Effect of Hydrogen Bonding on Entanglement Transition and Thermorheological Properties of Hyperbranched Polyglycerol Melts. Macromolecules 2016, 49, 8722-8737. 


\section{Graphical TOC Entry}

\section{Linear viscoelasticity of carbosilane dendrimer melts}

Maxim Dolgushev, Denis A. Markelov, and Erkki Lähderanta

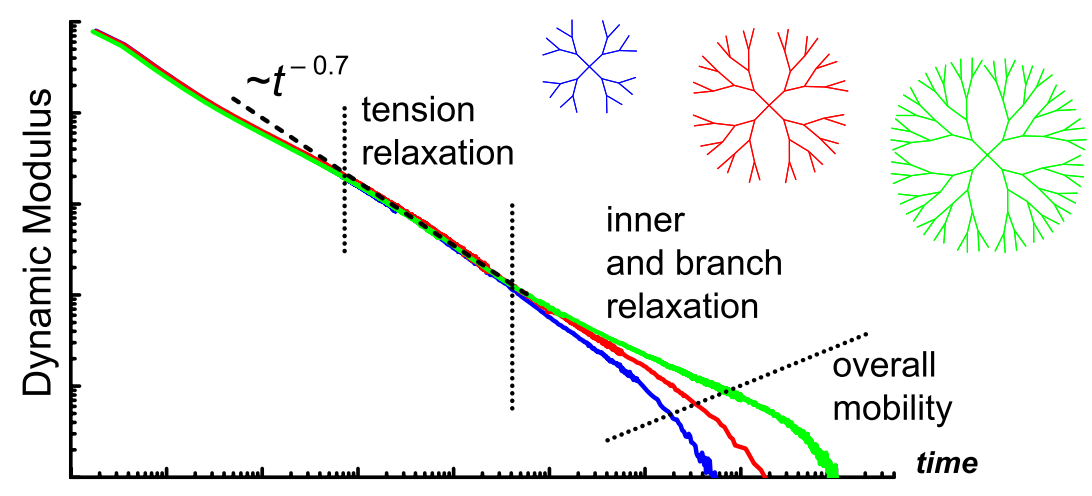

